\title{
Modified Eccentric Connectivity Index and Polynomial of Corona Product of Graphs
}

\author{
Nilanjan De \\ Department of Basic Sciences and Humanities, \\ Calcutta Institute of Engineering and Management, \\ Kolkata 700040, India
}

\author{
Sk. Md. Abu Nayeem \\ Department of Mathematics, \\ Aliah University, DN-20, Sector-V, \\ Salt Lake, Kolkata 700091, India
}

\author{
Anita Pal \\ Department of Mathematics, \\ National Institute of Technology \\ Durgapur 713209, India
}

\begin{abstract}
The eccentric connectivity index of a graph is defined as the sum of the products of eccentricity with the degree of vertices over all vertices of the graph, and the modified eccentric connectivity index of a graph is defined as the sum of the products of eccentricity with the total degree of neighbouring vertices over all vertices of the graph. In this study, we find eccentric connectivity index and modified eccentric connectivity index and their respective polynomial versions of corona product of two graphs. Finally, we calculate the eccentric connectivity index and modified eccentric connectivity index of some important classes of chemically interesting molecular graphs by specializing the components of corona product of graphs.
\end{abstract}

\section{Keywords}

Eccentricity, topological index, eccentric connectivity index, modified eccentric connectivity index, corona product

\section{INTRODUCTION}

Topological indices are real numbers derived from the molecular graph structure for correlation of chemical structure with various physical properties, chemical reactivity or biological activity and two graphs $G$ and $H$ have the equal value of a particular topological index if $G \cong H$. In chemistry, biochemistry and nanotechnology different topological indices are found to be useful in isomer discrimination, structure-property relationship, structure-activity relationship, pharmaceutical drug design and so forth. Various topological indices have been defined in chemical literature and various applications and mathematical properties of these indices have been found. The concept of topological indices was started when the American chemist Harold Wiener introduced the first topological index, named as Wiener index [1], in 1947 for investigating boiling points of alkanes. Suppose $G$ be a simple connected graph and $V(G)$ and $E(G)$ respectively denote the vertex set and edge set of $G$. Let, for any vertex $v \in V(G), d_{G}(v)$ denotes its degree, that is the number of neighbor of $v$ and $N(v)$ denotes the set of vertices which are the neighbors of the vertex $v$, so that $|N(v)|=d_{G}(v)$. Also let

$$
\delta_{G}(v)=\sum_{u \in N(v)} d_{G}(u)
$$

i.e., sum of degrees of the neighbor vertices of $G$. If $u, v \in V(G)$, then $d_{G}(u, v)$ is the minimum number of edges in the $(u, v)$-paths in $G$. Let $\varepsilon_{G}(v)$ denotes the eccentricity of a vertex $v$ and is defined as the largest distance from $v$ to any other vertex of $G$. Various vertex eccentricity and degree based topological indices are found in literature which is used to understand various properties of chemical compounds in theoretical chemistry. The total eccentricity index $\zeta(G)$ of a graph is defined as the sum of eccentricity of all the vertices of $G$ [2] 3]. The eccentric connectivity index of a graph $G$ is one of the most popular topological indices and is defined as [4]

$$
\xi^{c}(G)=\sum_{v \in V(G)} d_{G}(v) \varepsilon_{G}(v) .
$$

For various applications and for more results related to chemical applications and mathematical properties of this index, we refer our reader to [5, 6, 7, 8, 9]. One modified version of eccentric connectivity index is defined as [10]

$$
\xi_{c}(G)=\sum_{v \in V(G)} \delta_{G}(v) \varepsilon_{G}(v) .
$$

In [11] and [10], the modified eccentric connectivity polynomial for three infinite classes of fullerenes and one-pentagonal carbon nanocones was computed. In [12], some exact relations for the modified eccentric connectivity polynomial of different graph operations were derived. Different upper and lower bounds are obtained and some study on different generalized thorn graphs for this modified eccentric connectivity index is done by the present authors in [13] and [14]. The first Zagreb index of $G$ denoted by $M_{1}(G)$ is one of the oldest vertex degree based topological indices introduced in [15] by Gutman and Trinajstić and is defined as

$$
M_{1}(G)=\sum_{i=1}^{n} d_{G}\left(u_{i}\right)^{2}=\sum_{i=1}^{n} \delta_{G}\left(u_{i}\right) .
$$

Let $G_{1}$ and $G_{2}$ be two simple connected graphs with $n_{i}$ number of vertices and $e_{i}$ number of edges respectively, for $i \in\{1,2\}$. The corona product $G_{1} \circ G_{2}$ of these two graphs is obtained by taking one copy of $G_{1}$ and $n_{1}$ copies of $G_{2}$; and by joining each vertex of the $i$-th copy of $G_{2}$ to the $i$-th vertex of $G_{1}$, where $1 \leq i \leq n_{1}$. The corona product of $G_{1}$ and $G_{2}$ has total number of $\left(n_{1} n_{2}+n_{1}\right)$ vertices and $\left(e_{1}+n_{1} e_{2}+n_{1} n_{2}\right)$ edges. Clearly, the corona product operation of two graphs is not commutative. Different topological indices such as Wiener-type Indices [16], Szeged, vertex PI, first and second Zagreb indices [17], weighted PI index [18], etc. of the corona product of two graphs have already been studied. 
Corona product of graphs appears in chemical literature as plerographs of the hydrogen suppressed molecular graphs known as kenographs. In this study, we find eccentric connectivity index and modified eccentric connectivity index and their respective polynomial versions of corona product of two graphs. Furthermore, by specializing the components of corona product of graphs we calculate the eccentric connectivity index and modified eccentric connectivity index of $t$-thorny graph, sunlet graph, bottleneck graph, suspension of graphs and some classes of bridge graphs.

\section{MAIN RESULTS}

Let the vertices of $G_{1}$ are denoted by $V\left(G_{1}\right)=\left\{u_{1}, u_{2}, \ldots, u_{n_{1}}\right\}$ and the vertices of the $i$-th copy of $G_{2}$ are denoted by $V\left(G_{2, i}\right)=\left\{v_{1}^{i}, v_{2}^{i}, \ldots, v_{n_{2}}^{i}\right\}$ for $i=1,2, \ldots, n_{1}$. Thus the vertex set and edge set of $G_{1} \circ G_{2}$ are given by $V\left(G_{1} \circ G_{2}\right)=V\left(G_{1}\right) \bigcup_{i=1}^{n_{1}} V\left(G_{2, i}\right)$ and $E\left(G_{1} \circ G_{2}\right)=$ $E\left(G_{1}\right) \bigcup_{i=1}^{n_{1}} E\left(G_{2, i}\right) \bigcup\left\{\left(u_{i}, v_{j}^{i}\right): u_{i} \in V\left(G_{1}\right), v_{j}^{i} \in V\left(G_{2, i}\right)\right\}$.

First we start with following important lemma.

LEMMA 1. The degree, eccentricity and neighborhood degree sum of the vertices of $G_{1} \circ G_{2}$ are given as follows.

(a) If $u_{i} \in V\left(G_{1}\right)$, then
(i) $d_{G_{1} \circ G_{2}}\left(u_{i}\right)=d_{G_{1}}\left(u_{i}\right)+n_{2}$,
(ii) $\varepsilon_{G_{1} \circ G_{2}}\left(u_{i}\right)=\varepsilon_{G_{1}}\left(u_{i}\right)+1$, and
(iii) $\delta_{G_{1} \circ G_{2}}\left(u_{i}\right)=\delta_{G_{1}}\left(u_{i}\right)+n_{2} d_{G_{1}}\left(u_{i}\right)+2 m_{2}+n_{2}$.

(b) If $v_{j}^{i} \in V\left(G_{2, i}\right)$, where the $i$-th copy of $G_{2}$ is denoted by $G_{2, i}$, $1 \leq i \leq\left|V\left(G_{1}\right)\right|$, then

(i) $d_{G_{1} \circ G_{2}}\left(v_{j}^{i}\right)=d_{G_{2}}\left(v_{j}\right)+1$,

(ii) $\varepsilon_{G_{1} \circ G_{2}}\left(v_{j}^{i}\right)=\varepsilon_{G_{1}}\left(u_{i}\right)+2$, and

(iii) $\delta_{G_{1} \circ G_{2}}\left(v_{j}^{i}\right)=\delta_{G_{1}}\left(v_{j}\right)+d_{G_{2}}\left(v_{j}\right)+d_{G_{1}}\left(u_{i}\right)+n_{2}$.

In the following, we start by computing the eccentric connectivity index of corona product of two graphs.

THEOREM 1. The eccentric connectivity index of $G_{1} \circ G_{2}$ is given by

$\xi^{c}\left(G_{1} \circ G_{2}\right)=\xi^{c}\left(G_{1}\right)+2\left(n_{2}+m_{2}\right) \zeta\left(G_{1}\right)+2 m_{1}+4 n_{1} m_{2}+3 n_{1} n_{2}$

Proof. From the definition of corona product of graphs, the eccentric connectivity index of $G_{1} \circ G_{2}$ is given by

$$
\begin{aligned}
\xi^{c}\left(G_{1} \circ G_{2}\right)= & \sum_{i=1}^{n_{1}} d_{G_{1} \circ G_{2}}\left(u_{i}\right) \varepsilon_{G_{1} \circ G_{2}}\left(u_{i}\right) \\
& +\sum_{i=1}^{n_{1}} \sum_{j=1}^{n_{2}} d_{G_{1} \circ G_{2}}\left(v_{j}^{i}\right) \varepsilon_{G_{1} \circ G_{2}}\left(v_{j}^{i}\right) .
\end{aligned}
$$

Now, using $(a)(i)$ and $(a)(i i)$ of Lemma 1 we have

$$
\begin{aligned}
& \sum_{i=1}^{n_{1}} d_{G_{1} \circ G_{2}}\left(u_{i}\right) \varepsilon_{G_{1} \circ G_{2}}\left(u_{i}\right) \\
= & \sum_{i=1}^{n_{1}}\left\{d_{G_{1}}\left(u_{i}\right)+n_{2}\right\}\left\{\varepsilon_{G_{1}}\left(u_{i}\right)+1\right\}
\end{aligned}
$$

$$
\begin{aligned}
& =\sum_{i=1}^{n_{1}} d_{G_{1}}\left(u_{i}\right) \varepsilon_{G_{1}}\left(u_{i}\right)+n_{2} \sum_{i=1}^{n_{1}} \varepsilon_{G_{1}}\left(u_{i}\right)+\sum_{i=1}^{n_{1}} d_{G_{1}}\left(u_{i}\right)+n_{1} n_{2} \\
& =\xi^{c}\left(G_{1}\right)+n_{2} \zeta\left(G_{1}\right)+2 m_{1}+n_{1} n_{2} .
\end{aligned}
$$

Also, using $(b)(i)$ and $(b)(i i)$ of Lemma 1 we get

$$
\begin{aligned}
& \sum_{i=1}^{n_{1}} \sum_{j=1}^{n_{2}} d_{G_{1} \circ G_{2}}\left(v_{j}^{i}\right) \varepsilon_{G_{1} \circ G_{2}}\left(v_{j}^{i}\right) \\
= & \sum_{i=1}^{n_{1}} \sum_{j=1}^{n_{2}}\left\{d_{G_{2}}\left(v_{j}\right)+1\right\}\left\{\varepsilon_{G_{1}}\left(u_{i}\right)+2\right\} \\
= & \sum_{i=1}^{n_{1}} \varepsilon_{G_{1}}\left(u_{i}\right) \sum_{j=1}^{n_{2}} d_{G_{2}}\left(v_{j}\right)+n_{2} \sum_{i=1}^{n_{1}} \varepsilon_{G_{1}}\left(u_{i}\right) \\
& +2 n_{1} \sum_{j=1}^{n_{2}} d_{G_{2}}\left(v_{j}\right)+2 n_{1} n_{2} \\
= & \left(n_{2}+2 m_{2}\right) \zeta\left(G_{1}\right)+4 n_{1} m_{2}+2 n_{1} n_{2} .
\end{aligned}
$$

Adding the above two, we get the desired result.

Luo and Wu computed the eccentric connectivity index of corona product of graphs as a particular case of cluster product of graphs in [19], which coincides with Theorem 1] Also, Došlić et al. in [20] computed the Theorem 1 , when calculating the eccentric connectivity index of different graph operations.

The eccentric connectivity polynomial [21] and total eccentricity polynomial [12] of $G$ are respectively defined as

$$
\operatorname{ECP}(G, x)=\sum_{i=1}^{n} d_{G}\left(v_{i}\right) x^{\varepsilon_{G}\left(v_{i}\right)}
$$

and

$$
\theta(G, x)=\sum_{i=1}^{n} x^{\varepsilon_{G}\left(v_{i}\right)}
$$

respectively. It is easy to see that the eccentric connectivity index and the total eccentricity index of a graph can be obtained from the corresponding polynomials by evaluating their first derivatives at $x=1$. In the following result we use the same reasoning of Theorem 1 to derive exact relation of eccentric connectivity polynomial of corona product of two graphs in terms of eccentric connectivity polynomial and total eccentricity polynomial of the first graph.

THEOREM 2. The eccentric connectivity polynomial of $G_{1} \circ G_{2}$ is given by

$\operatorname{ECP}\left(G_{1} \circ G_{2}, x\right)=x \operatorname{ECP}\left(G_{1}, x\right)+x\left((1+x) n_{2}+2 m_{2} x\right) \theta\left(G_{1}, x\right)$.

In the next paragraph, we give an exact expression for the modified eccentric connectivity index of corona product of graphs.

THEOREM 3. The modified eccentric connectivity index of $G_{1} \circ$ $G_{2}$ is given by

$$
\begin{aligned}
\xi_{c}\left(G_{1} \circ G_{2}\right) & =\xi_{c}\left(G_{1}\right)+2 n_{2} \xi^{c}\left(G_{1}\right)+\left(n_{2}+n_{2}^{2}+4 m_{2}\right) \zeta\left(G_{1}\right) \\
& +M_{1}\left(G_{1}\right)+\left(\zeta\left(G_{1}\right)+2 n_{1}\right) M_{1}\left(G_{2}\right) \\
& +6 n_{1} m_{2}+6 n_{2} m_{1}+n_{1} n_{2}\left(2 n_{2}+1\right) .
\end{aligned}
$$


Proof. According to definition of corona product of graphs the modified eccentric connectivity index of $G_{1} \circ G_{2}$ is given by

$$
\begin{aligned}
\xi_{c}\left(G_{1} \circ G_{2}\right)= & \sum_{i=1}^{n_{1}} \delta_{G_{1} \circ G_{2}}\left(u_{i}\right) \varepsilon_{G_{1} \circ G_{2}}\left(u_{i}\right) \\
& +\sum_{i=1}^{n_{1}} \sum_{j=1}^{n_{2}} \delta_{G_{1} \circ G_{2}}\left(v_{j}^{i}\right) \varepsilon_{G_{1} \circ G_{2}}\left(v_{j}^{i}\right) .
\end{aligned}
$$

Now, using $(a)(i i)$ and $(a)(i i i)$ of Lemma 1 we have

$$
\begin{aligned}
& \sum_{i=1}^{n_{1}} \delta_{G_{1} \circ G_{2}}\left(u_{i}\right) \varepsilon_{G_{1} \circ G_{2}}\left(u_{i}\right) \\
= & \sum_{i=1}^{n_{1}}\left\{\delta_{G_{1}}\left(u_{i}\right)+n_{2} d_{G_{1}}\left(u_{i}\right)+2 m_{2}+n_{2}\right\}\left\{\varepsilon_{G_{1}}\left(u_{i}\right)+1\right\} \\
= & \sum_{i=1}^{n_{1}} \delta_{G_{1}}\left(u_{i}\right) \varepsilon_{G_{1}}\left(u_{i}\right)+n_{2} \sum_{i=1}^{n_{1}} d_{G_{1}}\left(u_{i}\right) \varepsilon_{G_{1}}\left(u_{i}\right) \\
& +\left(2 m_{2}+n_{2}\right) \sum_{i=1}^{n_{1}} \varepsilon_{G_{1}}\left(u_{i}\right)+\sum_{i=1}^{n_{1}} \delta_{G_{1}}\left(u_{i}\right) \\
& +n_{2} \sum_{i=1}^{n_{1}} d_{G_{1}}\left(u_{i}\right)+\left(2 m_{2}+n_{2}\right) n_{1} \\
= & \xi_{c}\left(G_{1}\right)+n_{2} \xi^{c}\left(G_{1}\right)+\left(2 m_{2}+n_{2}\right) \zeta\left(G_{1}\right)+M_{1}\left(G_{1}\right) \\
& +2 n_{2} m_{1}+n_{1}\left(2 m_{2}+n_{2}\right) .
\end{aligned}
$$

Similarly using $b(i i)$ and $b(i i i)$ of Lemma 1 , we get

$$
\begin{aligned}
& \sum_{i=1}^{n_{1}} \sum_{j=1}^{n_{2}} \delta_{G_{1} \circ G_{2}}\left(v_{j}^{i}\right) \varepsilon_{G_{1} \circ G_{2}}\left(v_{j}^{i}\right) \\
= & \sum_{i=1}^{n_{1}} \sum_{j=1}^{n_{2}}\left\{\delta_{G_{1}}\left(v_{j}\right)+d_{G_{2}}\left(v_{j}\right)+d_{G_{1}}\left(u_{i}\right)+n_{2}\right\}\left\{\varepsilon_{G_{1}}\left(u_{i}\right)+2\right\} \\
= & \sum_{i=1}^{n_{1}} \varepsilon_{G_{1}}\left(u_{i}\right) \sum_{j=1}^{n_{2}} \delta_{G_{2}}\left(v_{j}\right)+\sum_{i=1}^{n_{1}} \varepsilon_{G_{1}}\left(u_{i}\right) \sum_{i=1}^{n_{2}} d_{G_{2}}\left(v_{j}\right) \\
& +n_{2}^{2} \sum_{i=1}^{n_{1}} \varepsilon_{G_{1}}\left(u_{i}\right)+2 \sum_{i=1}^{n_{1}} \sum_{j=1}^{n_{2}} \delta_{G_{2}}\left(v_{j}\right)+2 \sum_{i=1}^{n_{1}} \sum_{j=1}^{n_{2}} d_{G_{2}}\left(v_{j}\right) \\
& +n_{2} \sum_{i=1}^{n_{1}} d_{G_{1}}\left(u_{i}\right) \varepsilon_{G_{1}}\left(u_{i}\right)+2 n_{2} \sum_{i=1}^{n_{1}} d_{G_{1}}\left(u_{i}\right)+2 n_{1} n_{2}^{2} \\
= & \zeta\left(G_{1}\right) M_{1}\left(G_{2}\right)+2 m_{2} \zeta\left(G_{1}\right)+n_{2} \xi^{c}\left(G_{1}\right)+n_{2}{ }^{2} \zeta\left(G_{1}\right) \\
& +2 n_{1} M_{1}\left(G_{2}\right)+4 n_{1} m_{2}+4 n_{2} m_{1}+2 n_{1} n_{2}{ }^{2} .
\end{aligned}
$$

The expression for the modified eccentric connectivity index of $G_{1} \circ G_{2}$ follows by summing the above two expressions.

The modified eccentric connectivity polynomial of $G$ is defined as [11, 10, 12]

$$
\operatorname{MECP}(G, x)=\sum_{i=1}^{n} \delta_{G}\left(v_{i}\right) x^{\varepsilon_{G}\left(v_{i}\right)}
$$

so that, the modified eccentric connectivity index of a graph can be obtained from this polynomial by evaluating its first derivative at $x=1$. In the following we give the modified eccentric connectivity polynomial of $G_{1} \circ G_{2}$ using the similar arguments as Theorem 3

THEOREM 4. The modified eccentric connectivity polynomial of $G_{1} \circ G_{2}$ is given by

$$
\begin{aligned}
\operatorname{MECP}\left(G_{1} \circ G_{2}, x\right)= & x M E C P\left(G_{1}, x\right)+x(1+x) n_{2} \operatorname{ECP}\left(G_{1}, x\right) \\
& +x\left(x M_{1}\left(G_{2}\right)+2(1+x) m_{2}\right. \\
& \left.+n_{2}\left(n_{2} x+1\right)\right) \theta\left(G_{1}, x\right) .
\end{aligned}
$$

\section{COROLLARIES AND EXAMPLES}

For any given graph $G$, the $t$-thorny graph or the $t$-fold bristled graph is obtained by attaching $t$-vertices of degree one to each vertex of $G$. This graph was introduced by Gutman in [22] and can be obtained as corona product of $G$ and complement of complete graph on $t$-vertices, i.e., $\overline{K_{t}}$. Let $G$ be a connected graph with $n$ vertices and $m$ edges. In the following we find the eccentric connectivity index of $t$-thorny graphs from eccentric connectivity index of corona product of graphs. For different topological indices of thorny graphs interested reader may refer to [21, 23, 14].

COROLlary 1. The eccentric connectivity index of $G^{t}$ is given by

$$
\xi^{c}\left(G^{t}\right)=\xi^{c}(G)+2 t \zeta(G)+2 m+3 n t .
$$

Proof. This result is a straight forward application of Theorem 1. $\square$

Total eccentricity indices of $P_{n}(n \geq 1)$ and $C_{n}(n \geq 3)$, i.e., path and cycle of order $n$ respectively, are given by

$$
\begin{gathered}
\zeta\left(P_{n}\right)= \begin{cases}\frac{3}{4} n^{2}-\frac{1}{2} n, & \text { when } n \text { is even } \\
\frac{3}{4} n^{2}-\frac{1}{2} n-\frac{1}{4}, & \text { when } n \text { is odd, }\end{cases} \\
\zeta\left(C_{n}\right)= \begin{cases}\frac{1}{2} n^{2}, & \text { when } n \text { is even } \\
\frac{1}{2} n(n-1), & \text { when } n \text { is odd. }\end{cases}
\end{gathered}
$$

and the eccentric connectivity indices are given by

$$
\begin{gathered}
\xi^{c}\left(P_{n}\right)= \begin{cases}\frac{1}{2}\left(3 n^{2}-6 n+4\right), & \text { when } n \text { is even } \\
\frac{3}{2}(n-1)^{2}, & \text { when } n \text { is odd, }\end{cases} \\
\xi^{c}\left(C_{n}\right)= \begin{cases}n^{2}, & \text { when } n \text { is even } \\
n(n-1), & \text { when } n \text { is odd. }\end{cases}
\end{gathered}
$$

Then from (5) the following result follows.

EXAMPLE 1. The eccentric connectivity index of t-thorny graph of $C_{n}$ and $P_{n}$ are given by

(i)

$$
\xi^{c}\left(C_{n} \circ \overline{K_{t}}\right)= \begin{cases}n^{2}(t+1)+n(3 t+2), & \text { when } n \text { is even } \\ n(n-1)(t+1)+n(3 t+2), & \text { when } n \text { is odd } .\end{cases}
$$

(ii)

$$
\xi^{c}\left(P_{n} \circ \overline{K_{t}}\right)= \begin{cases}\frac{3}{2} n^{2}(t+1)+2 n t-n, & \text { when } n \text { is even } \\ \frac{3}{2} n^{2}(t+1)+2 n t-n & \\ -\frac{1}{2} t-\frac{1}{2}, & \text { when } n \text { is odd },\end{cases}
$$


Similar to eccentric connectivity index, the modified eccentric connectivity index of $t$-thorny graph is obtained from (3) as follows.

COROLlary 2. The modified eccentric connectivity index of $G^{t}$ is given by

$\xi_{c}\left(G^{t}\right)=\xi_{c}(G)+2 t \xi^{c}(G)+t(t+1) \zeta(G)+M_{1}(G)+6 m t+n t+2 n t^{2}$.

The modified eccentric connectivity index of $P_{n}(n \geq 4)$ and $C_{n}(n \geq 3)$ are respectively given by

$$
\begin{gathered}
\xi_{c}\left(P_{n}\right)= \begin{cases}3 n^{2}-8 n+8, & \text { when } n \text { is even } \\
3 n^{2}-8 n+7, & \text { when } n \text { is odd, }\end{cases} \\
\xi_{c}\left(C_{n}\right)= \begin{cases}2 n^{2}, & \text { when } n \text { is even } \\
2 n(n-1), & \text { when } n \text { is odd. }\end{cases}
\end{gathered}
$$

Then the following result follows from 6 .

EXAMPLE 2. The modified eccentric connectivity index of $t$ thorny graph of $C_{n}$ and $P_{n}$ are given by

(i)

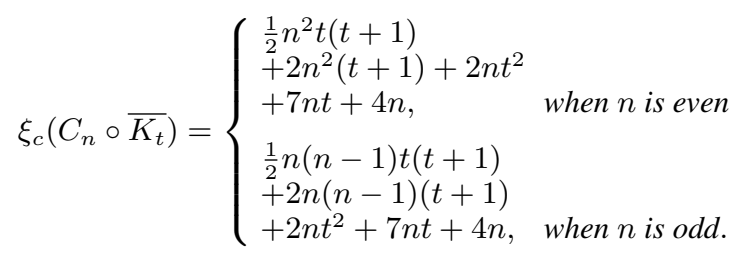

(ii)

$$
\xi_{c}\left(P_{n} \circ \overline{K_{t}}\right)= \begin{cases}\frac{3}{4} n^{2} t^{2}+\frac{3}{2} n t^{2}+\frac{15}{4} n^{2} t & \\ +\frac{1}{2} n t+3 n^{2}-4 n & \\ -2 t+2, & \text { when } n \text { is even } \\ \frac{3}{4} n^{2} t^{2}+\frac{3}{2} n t^{2}+\frac{15}{4} n^{2} t & \\ +\frac{1}{2} n t+3 n^{2}-4 n-\frac{1}{4} t^{2} & \\ -\frac{13}{4} t+1, & \text { when } n \text { is odd } .\end{cases}
$$

It can be easily checked that the formula for eccentric connectivity index and modified eccentric connectivity index of $t$-thorny graph coincides with the results directly derived in [21] and [14] respectively.

A particular thorny graph, the $n$-sunlet graph is obtained by attaching $n$ pendent edges to the cycle $C_{n}$, so that it contains $2 n$ vertices and edges. Let it be denoted by $S L_{n}$ and thus $S L_{n}=C_{n} \circ \overline{K_{1}}$. So using (1) and (3), the following result follows.

EXAMPLE 3. The eccentric connectivity index and modified eccentric connectivity index of $S L_{n}(n \geq 3)$, is given by (i)

(ii)

$$
\xi^{c}\left(S L_{n}\right)= \begin{cases}2 n^{2}+5 n, & \text { when } n \text { is even } \\ 2 n(n-1)+5 n, & \text { when } n \text { is odd }\end{cases}
$$

$$
\xi_{c}\left(S L_{n}\right)=\left\{\begin{array}{l}
5 n^{2}+13 n, \text { when } n \text { is even } \\
5 n^{2}+8 n, \text { when } n \text { is odd } .
\end{array}\right.
$$

A bistar $B_{n, n}$ is obtained by joining the center vertices of two copies of $K_{1, n}$. Thus the star graph $S_{n}$ on $n$ vertices and the bistar $B_{n, n}$ on $(2 n+2)$ vertices are corona product of $K_{1}$ and $\overline{K_{n-1}}$, and $P_{2}$ and $\overline{K_{n}}$ respectively.
EXAMPLE 4. The eccentric connectivity index and modified eccentric connectivity index of star graph is given by $\xi^{c}\left(K_{1} \circ\right.$ $\left.\overline{K_{n-1}}\right)=3(n-1)$ and $\xi_{c}\left(K_{1} \circ \overline{K_{n-1}}\right)=2 n^{2}-3 n+1$.

EXAMPLE 5. The eccentric connectivity index and modified eccentric connectivity index of bistar $B_{n, n}$ is given by $\xi^{c}\left(P_{2} \circ\right.$ $\left.\overline{K_{n}}\right)=10 n+4$ and $\xi_{c}\left(P_{2} \circ \overline{K_{n}}\right)=6 n^{2}+14 n+4$.

The suspension of a graph $G$ is defined as corona product of $K_{1}$ and $G$. So from (1) and (3) the following result follows.

COROLlARY 3. The eccentric connectivity index and modified eccentric connectivity index of suspension of $G$ are respectively given by $\xi^{c}\left(K_{1} \circ G\right)=3 n+4 m$ and $\xi_{c}\left(K_{1} \circ G\right)=$ $2 M_{1}(G)+2 n^{2}+n+6 m$.

EXAMPLE 6. The wheel graph $W_{n}$ on $(n+1)$ vertices is the suspension of $C_{n}$. So its eccentric connectivity index and modified eccentric connectivity index are given by $\xi^{C}\left(K_{1} \circ C_{n}\right)=7 n$ and $\xi_{c}\left(K_{1} \circ C_{n}\right)=2 n^{2}+15 n$.

EXAMPLE 7. The fan graph $F_{n}$ on $(n+1)$ vertices is the suspension of $P_{n}$. So its eccentric connectivity index and modified eccentric connectivity index are given by $\xi^{c}\left(K_{1} \circ P_{n}\right)=7 n-4$ and $\xi_{c}\left(K_{1} \circ P_{n}\right)=2 n^{2}+15 n-18$.

The bottleneck graph $B$ can be obtained by the corona product of $K_{2}$ and $G$. So from (1) and (3) the following result follows.

COROLLARY 4. The eccentric connectivity index and modified eccentric connectivity index of the graph $B$ is given by

(i) $\xi^{c}(B)=10 n+12 m+4$

(ii) $\xi_{c}(B)=6 M_{1}(G)+6 n^{2}+14 n+20 m+4$.

Let $v_{1}, v_{2}, \ldots, v_{n}$ be the vertices of a set of finite pair wise disjoint graphs $G_{1}, G_{2}, \ldots, G_{n}$ respectively. Then the bridge graph with respect to the vertices $v_{1}, v_{2}, \ldots, v_{n}$ is denoted by $B\left(G_{1}, G_{2}, \ldots, G_{n} ; v_{1}, v_{2}, \ldots, v_{n}\right)$ which is obtained by joining the vertices $v_{i}$ and $v_{i+1}$ of $G_{i}$ and $G_{i+1}$ by an edge, for all $i=$ $1,2, \ldots,(n-1)$. In particular, if $G_{1} \cong G_{2} \cong \ldots \cong G_{n} \cong G$ and $v_{1}=v_{2}=\ldots=v_{n}=v$, then the bridge graph is denoted by $G_{n}(G, v)$. Now we consider three particular type of bridge graphs as in [24, 2, 18], named as $B_{n}=G_{n}\left(P_{3}, v\right), T_{m, k}=G_{m}\left(C_{k}, u\right)$ and $J_{n, m+1}=G_{n}\left(P_{3}, v\right)$. According to definition of corona product of graphs the bridge graphs $B_{n}=P_{n} \circ \overline{K_{2}}, T_{m, 3}=P_{m} \circ K_{2}$ and $J_{n, m+1}=P_{n} \circ C_{m}$. So using, 11 we obtain the eccentric connectivity index of these bridge graphs.

EXAMPLE 8. (i) For $n \geq 2$,

$$
\xi^{c}\left(B_{n}\right)= \begin{cases}\frac{9}{2} n^{2}+3 n, & \text { when } n \text { is even } \\ \frac{9}{2} n^{2}+3 n-\frac{3}{2}, & \text { when } n \text { is odd } .\end{cases}
$$

(ii) For $m \geq 2$,

$$
\xi^{c}\left(T_{m, 3}\right)= \begin{cases}6 m^{2}+6 m, & \text { when } m \text { is even } \\ 6 m^{2}+6 m-2, & \text { when } m \text { is odd } .\end{cases}
$$

(iii) For $n \geq 2$ and $m \geq 3$,

$$
\xi^{c}\left(J_{n, m+1}\right)=\left\{\begin{array}{l}
3 n^{2}\left(m+\frac{1}{2}\right)+5 m n-n, \text { when } n \text { is even } \\
3 n^{2}\left(m+\frac{1}{2}\right)+5 m n-n \\
-m-\frac{1}{2}, \quad \text { when } n \text { is odd } .
\end{array}\right.
$$

Similarly, using (3), the modified eccentric connectivity index of the bridge graphs $P_{n} \circ \bar{K}_{2}, P_{m} \circ K_{2}$ and $P_{n} \circ C_{m}$ are obtained as follows. 
EXAMPLE 9. (i) For $n \geq 3$,

$$
\xi_{c}\left(B_{n}\right)= \begin{cases}\frac{27}{2} n^{2}+3 n-2, & \text { when } n \text { is even } \\ \frac{27}{2} n^{2}+3 n-\frac{13}{2}, & \text { when } n \text { is odd } .\end{cases}
$$

(ii) For $m \geq 3$,

$$
\xi_{c}\left(T_{m, 3}\right)=\left\{\begin{array}{l}
18 m^{2}+10 m-2, \text { when } m \text { is even } \\
18 m^{2}+10 m-8, \text { when } m \text { is odd } .
\end{array}\right.
$$

(iii) For $n \geq 3$ and $m \geq 3$,

$$
\xi_{c}\left(J_{n, m+1}\right)=\left\{\begin{array}{l}
3 n^{2}+3 m n^{2}+2 n m^{2} \\
+15 n m-4 n-2 m+2 \\
+\left(\frac{3}{4} n^{2}-\frac{1}{2} n\right)\left(m^{2}+9 m\right), \quad \text { if } n \text { is even } \\
3 n^{2}+3 m n^{2}+2 n m^{2} \\
+15 n m-4 n-3 m+1 \\
+\left(\frac{3}{4} n^{2}-\frac{1}{2} n-\frac{1}{4}\right)\left(m^{2}+9 m\right), \text { if } n \text { is odd. }
\end{array}\right.
$$

\section{CONCLUSION}

In this study, we have computed the eccentric connectivity index and modified eccentric connectivity index and their respective polynomials for corona product of graphs. Also, we apply our results to calculate these indices for some classes of graphs such as $t$-thorny graph, sunlet graph, bottleneck graph, suspension of graphs and bridge graphs by considering corona product of specific graphs. Nevertheless, there are still many classes of graphs which are not covered in this study. Various chemical graphs and nano-structure may also be considered and evaluation of these indices in terms of degeneracy, discriminating power and intercorrelation for such classes of graphs can be further investigated in this direction.

\section{REFERENCES}

[1] H. Wiener, Structural determination of paraffin boiling points, Journal of American Chemical Society, 69(1)(1947), 17-20.

[2] N. De, S. M. A. Nayeem and A. Pal, Total eccentricity index of the generalized hierarchical product of graphs, International Journal of Applied and Computational Mathematics, 1(2014), doi: 10.1007/s40819-014-0016-4.

[3] K. Fathalikhani, H. Faramarzi and H. Yousefi-Azari, Total eccentricity of some graph operations, Electronic Notes in Discrete Math., 45(2014), 125-131.

[4] V. Sharma, R. Goswami and A. K. Madan, Eccentric connectivity index: A novel highly discriminating topological descriptor for structure-property and structure-activity studies, Journal of Chemical Information and Modeling, 37 (1997) 273-282.

[5] T. Došlić, M. Saheli and D. Vukićević, Eccentric connectivity index: extremal graphs and Values, Iranian Journal of Mathematical Chemistry, 1(2010), 45-56.

[6] T. Došlić, A. Graovac and O. Ori, Eccentric connectivity index of hexagonal belts and chains, MATCH Communications in Mathematical and in Computer Chemistry, 65(2011), 745752.

[7] B. Eskender and E. Vumar, Eccentric connectivity index and eccentric distance sum of some graph operations, Transactions on Combinatorics, 2(1)(2013), 103-111.

[8] A. Ilić and I. Gutman, Eccentric connectivity index of chemical trees, MATCH Communications in Mathematical and in Computer Chemistry, 65(2011), 731-744.
[9] B. Zhou and Z. Du, On eccentric connectivity index, $M A T C H$ Communications in Mathematical and in Computer Chemistry, 63 (2010), 181-198.

[10] A. R. Ashrafi, M. Ghorbani, A Study of fullerenes by MEC polynomials, Electronic Materials Letters, 6(2)(2010), 8790.

[11] M. Alaeiyan, J. Asadpour, R. Mojarad, A numerical method for MEC polynomial and MEC index of one-pentagonal carbon nanocones, Fullerenes, Nanotubes and Carbon Nanostructures, 21(10)(2013), 825-835.

[12] A. R. Ashrafi, M. Ghorbani and M. A. Hossein-Zadeh, The eccentric connectivity polynomial of some graph operations, Serdica Journal of Computing, 5(2011), 101-116.

[13] N. De, S. M. A. Nayeem and A. Pal, Bounds for modified eccentric connectivity index, Advanced Modeling and Optimization, 16(1)(2014), 133-142.

[14] N. De, S. M. A. Nayeem and A. Pal, Modified Eccentric Connectivity of Generalized Thorn Graphs, International Journal of Computational Mathematics, 2014(2014), doi: $10.1155 / 2014 / 436140$.

[15] I. Gutman and N. Trinajstić, Graph theory and molecular orbitals: Total $\varphi$-electron energy of alternant hydrocarbons, Chemical Physics Letters, 17(1972), 535-538.

[16] H. Bian, X. Ma, and E. Vumar, The Wiener-type indices of the corona of Two graphs, Ars Combinatoria, 107 (2012), 193199.

[17] Z. Yarahmadi and A. R. Ashrafi, The Szeged, Vertex PI, first and second Zagreb Indices of corona Product of Graphs, Filomat, 26(3)(2012), 467-472.

[18] K. Pattabiraman and P. Kandan, Weighted PI index of corona product of graphs, Discrete Mathematics, Algorithms and Applications, 06(2014), doi: 10.1142/S1793830914500554.

[19] Z. Luo and J. Wu, Zagreb eccentricity indices of the generalized hierarchical product graphs and their applications, Journal of Applied Mathematics, 2014(2014), doi: 10.1155/2014/241712.

[20] T. Doslic and M. Saheli,Eccentric connectivity index of composite graphs, Utilitas Mathematica, 95 (2014), 3-22.

[21] N. De, On eccentric connectivity index and polynomial of thorn graph, Applied Mathematics, 3(2012), 931-934.

[22] I. Gutman, Distance in thorny graph, Publications de l'Institut Mathmatique (Beograd), 63(1998), 31-36.

[23] N. De, Augmented eccentric connectivity index of some thorn graphs, International Journal of Applied Mathematical Research , 1(4) (2012) 671-680.

[24] Y. Alizadeh, A. Iranmanesh, T. Doslic and M. Azari, The edge Wiener index of suspensions, bottlenecks, and thorny graphs, Glasnik Matematicki Series III, 49(69)(2014), 1-12. 